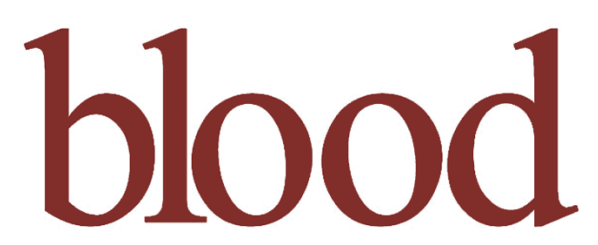

2005 106: 2356-2362

Prepublished online Jun 14, 2005;

doi:10.1182/blood-2005-02-0723

\title{
The TUBB1 Q43P functional polymorphism reduces the risk of cardiovascular disease in men by modulating platelet function and structure
}

Kathleen Freson, Rita De Vos, Christine Wittevrongel, Chantal Thys, Johan Defoor, Luc Vanhees, Jos Vermylen, Kathelijne Peerlinck and Chris Van Geet

Updated information and services can be found at:

http://bloodjournal.hematologylibrary.org/cgi/content/full/106/7/2356

Articles on similar topics may be found in the following Blood collections:

Hemostasis, Thrombosis, and Vascular Biology (2496 articles)

Cytoskeleton (143 articles)

Clinical Trials and Observations (2710 articles)

Information about reproducing this article in parts or in its entirety may be found online at:

http://bloodjournal.hematologylibrary.org/misc/rights.dtl\#repub_requests

Information about ordering reprints may be found online at:

http://bloodjournal.hematologylibrary.org/misc/rights.dtl\#reprints

Information about subscriptions and ASH membership may be found online at:

http://bloodjournal.hematologylibrary.org/subscriptions/index.dtl

Blood (print ISSN 0006-4971, online ISSN 1528-0020), is published semimonthly by the American Society of Hematology, 1900 M St, NW, Suite 200, Washington DC 20036.

Copyright 2007 by The American Society of Hematology; all rights reserved.

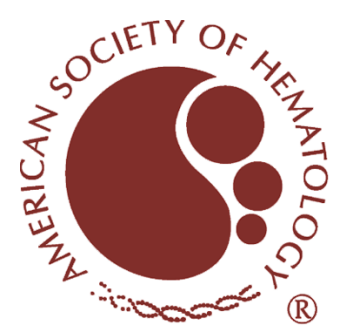




\section{The TUBB1 Q43P functional polymorphism reduces the risk of cardiovascular disease in men by modulating platelet function and structure}

Kathleen Freson, Rita De Vos, Christine Wittevrongel, Chantal Thys, Johan Defoor, Luc Vanhees, Jos Vermylen, Kathelijne Peerlinck, and Chris Van Geet

The discoid form of platelets is maintained by a marginal band of tightly coiled microtubules. $\beta 1$-tubulin is the major isoform within platelet and megakaryocyte microtubules. In $24.2 \%$ of 33 unrelated inherited macrothrombocytopenia patients and in $10.6 \%$ of 272 subjects of a healthy population a $P$ for $Q$ substitution in $\beta 1$-tubulin was found in the highly conserved residue 43 . Heterozygous carriers of the Q43P variant showed a reduced platelet protein $\beta 1$-tubulin expression. Transfection of green fluorescent protein (GFP)-tagged Q43P $\beta 1$-tubulin in megakaryocytic MEG01 cells resulted in a disturbed tubulin organization. Electron microscopy revealed enlarged spherocytic platelets with a disturbed marginal band and organelle-free zones. In addition, platelets with the Q43P $\beta 1$-tubulin variant had reduced adenosine triphosphate (ATP) secretion, thrombin receptor activating peptide (TRAP)-induced aggregation and collagen adhesion. The prevalence of the Q43P $\beta 1$ tubulin variant was also 2 times higher (odds ratio, $[O R]=2.1 ; 95 \%$ confidence interval $[\mathrm{Cl}], 1.22-3.59)$ among control subjects than among patients with cardiovascular disease (10.4\% versus $5.2 \%, P<.001$ ). By analyzing this protective factor in men and women separately, this association was only found in men. This study thus presents the functional consequences of the platelet Q43P $\beta 1$-tubulin substitution that is frequent in the healthy population and may protect men against arterial thrombosis. (Blood. 2005; 106:2356-2362)

() 2005 by The American Society of Hematology

\section{Introduction}

The $\alpha \beta$-tubulin heterodimer is the principal structural subunit of the microtubules, which are an important component of the surface membrane cytoskeleton and, together with the cytoplasmic, actinrich cytoskeleton, are responsible for intracellular transport of vesicles, cell morphogenesis, and chromosome segregation during cell division in all eukaryotes. ${ }^{1}$ Early structural studies already suggested tubulin heterogeneity, leading to the concept that various $\alpha$ - and $\beta$-tubulin isoforms are important determinants of tissuedependent functional differences among microtubules. ${ }^{2}$ The $\alpha$ - and $\beta$-tubulin isoforms contribute to the functional diversity of microtubules either through their differential polymerization, or by virtue of unique interactions with distinct microtubule-associated proteins (MAPs). Many studies have shown that the $\beta$-tubulin isoforms have unique biologic characteristics but are only partially interchangeable. ${ }^{3,4}$ There are at least 5 different $\beta$-tubulins in the mouse with $95 \%-97 \%$ homology, except for $\beta 1$-tubulin, which shares only about $78 \%$ identity with widely expressed tubulins such as $\beta 2$ - and $\beta 5$-tubulin. ${ }^{5}$ The amino acid differences between $\beta 1$-tubulin and other $\beta$-tubulins are scattered throughout the protein but are mainly concentrated in the carboxyl terminal region, which is located on the outer surface of the microtubules and interacts with MAPs. The $\beta 1$-tubulin residues involved in guanosine triphosphate (GTP) binding, an essential component for the regulation of microtubule assembly and function, are con- served. ${ }^{5} \beta 1$-tubulin is specifically expressed in platelets and mature megakaryocytes..$^{5-7}$

Platelet production by megakaryocytes is a poorly understood event, wherein differentiated megakaryocytes extrude long cytoplasmic processes ("proplatelets") that serve as the immediate precursors of circulating platelets. The microtubule cytoskeleton plays a central role in thrombopoiesis, ${ }^{8}$ and recent evidence suggests that platelet assembly occurs de novo within proplatelet extensions. ${ }^{9}$ $\beta 1$-tubulin is the predominant isoform in proplatelets and platelet marginal bands. In humans, the $\beta 1$-tubulin gene (TUBB1) is located on chromosome 20q13.3. Mice deficient in $\beta 1$-tubulin develop moderate thrombocytopenia as a result of reduced proplatelet formation and their spherocytic platelets carry a structurally defective marginal band and a reduced microtubule content. ${ }^{7,10}$ Although these $\beta 1$-tubulin knock-out mice have a prolonged bleeding time, their spherocytic platelets seem to give only minor disturbances of hemostasis in vitro and in vivo. ${ }^{7,10}$ The latter could be explained by the fact that the loss of $\beta 1$-tubulin in the platelets of these mice is partially compensated by the overexpression of alternate $\beta$-tubulin isoforms. ${ }^{7}$

At this moment, there are no functional studies on the role of $\beta 1$-tubulin in human platelets, nor is there evidence for a human $\beta 1$-tubulin-associated pathology. Here, we describe the first human $\beta 1$-tubulin functional substitution (Q43P) in a highly conserved
From the Center for Molecular and Vascular Biology, Department of Pathology, Cardiovascular Rehabilitation Unit, Department of Rehabilitation Sciences, Faculty of Physical Education and Physical Therapy, and Department of Pediatrics, University of Leuven, Belgium.

Submitted February 23, 2005; accepted June 5, 2005. Prepublished online as Blood First Edition Paper, June 14, 2005; DOI 10.1182/blood-2005-02-0723.

Supported by research grants G.0453.05 and G.0124.02 from the Fonds voor Wetenschappelijk Onderzoek Vlaanderen (Belgium) and GOA/2003/09 and OT/01/46 from Research Council of the University of Leuven (Onderzoeksraad K. U. Leuven, Belgium)
An Inside Blood analysis of this article appears at the front of this issue.

The online version of this article contains a data supplement

Reprints: Kathleen Freson, Center for Molecular and Vascular Biology, University of Leuven, Herestraat 49, B-3000 Leuven, Belgium; e-mail: kathleen.freson@med.kuleuven.be.

The publication costs of this article were defrayed in part by page charge payment. Therefore, and solely to indicate this fact, this article is hereby marked "advertisement" in accordance with 18 U.S.C. section 1734.

(C) 2005 by The American Society of Hematology 
residue, which exists in $10.6 \%$ of the general population and in $24.2 \%$ of 33 unrelated macrothrombocytopenia patients. We describe the influence of the Q43P $\beta$ 1-tubulin variant on platelet morphology and function. Since the $\beta 1$-tubulin variant reduces platelet reactivity, we hypothesized that the prevalence of this functional polymorphism should be lower in a patient population suffering from cardiovascular atherothrombotic disease.

\section{Patients, materials, and methods}

\section{Electron microscopy of platelets}

Platelet-rich fractions were available from all macrothrombocytopenia patients, 3 unrelated Q43P $\beta$ 1-tubulin carriers from a healthy population (carriers 1,2, and 4) and from 3 healthy noncarrier controls. Platelet-rich plasma (PRP) was prepared by centrifugation ( 15 minutes at $150 \mathrm{~g}$ ) of whole blood anticoagulated with $3.8 \%$ (wt/vol) trisodium citrate $(9: 1)$. The platelet-rich fractions were immediately fixed in $2.5 \%$ glutaraldehyde, 0.1 $\mathrm{M}$ phosphate buffer at $4^{\circ} \mathrm{C}$ overnight. After 1 hour postfixing in $1 \%$ osmium tetroxide, $0.1 \mathrm{M}$ phosphate buffer at $4^{\circ} \mathrm{C}$, the samples were dehydrated in graded series of alcohol and embedded in epoxy resin. Ultrathin sections were cut, stained with uranyl acetate-lead citrate, and examined using a Zeiss electron microscope (Heidelberg, Germany). ${ }^{11}$

\section{Genetic analysis and genotyping of TUBB1}

$\beta 1$-tubulin cDNA was amplified using primers $\beta 1$-tubulin1-forward (5'TCTCAGACTTGGGCTCAGAGC) and $\beta 1$-tubulin2-reverse (5'-TCCGCGGAAGCTTCTCTCAC). Genomic DNA was isolated from peripheral blood according to standard procedures. The cag to ccc mutation at nucleotide 129 of the human $\beta 1$-tubulin exon 2 was detected by digestion of polymerase chain reaction (PCR) products with restriction enzyme PvuII (Fermentas, St Leon-Rot, Germany). The following primers were used: $\beta 1$-tubulin3-forward (5'-AGTTCTGGGAGATGATTGGTG) and $\beta 1$ tubulin4-reverse (5'-ACCGTAGGCTTCGTTGTAGTAC) (detailed PCR conditions available upon request). Digested PCR fragments were separated on 3\% agarose gel and unrestricted PCR products have a size of 117 base pair (bp), while restriction generates bands of $74 \mathrm{bp}$ and $43 \mathrm{bp}$.

\section{GFP $\beta 1$-tubulin expression}

The wild-type and Q43P $\beta 1$-tubulin genes were cloned in the plasmid vector red-shifted green fluorescence protein (pEGFP)-C1 vector (Clontech, Palo Alto, CA). Transfection of MEG01 megakaryocytic cells was achieved by using the Fugene- 6 transfection reagent (Roche Diagnostics, Basel, Switzerland) according to the manufacturer's instructions. MEG01 cells were analyzed by immunofluorescence 48 hours after transfection, using an inverted epifluorescence microscope (Diaphot; Nikon, Melville, $\mathrm{NY}$ ), coupled to a charge-coupled device video camera (COHU, San Diego, CA). MEG01 adhesion studies were performed after coating glass coverslips with $200 \mu \mathrm{g} / \mathrm{mL}$ Horm collagen (Nycomed Arzenmittel, Munich, Germany) or with $20 \mu \mathrm{g} / \mathrm{mL}$ human fibrinogen (Sigma Chemical, St Louis, MO) for 16 hours at $4{ }^{\circ} \mathrm{C}$ and 2 washing steps with phosphate-buffered saline (PBS). MEG01 cells in PBS were incubated on collagen- or fibrinogen-coated coverslips for 45 minutes at $37^{\circ} \mathrm{C}$. Non-adherent cells were washed away and spreading of adherent transfected MEG01 cells were observed under a fluorescent microscope (Zeiss LSM 510 confocal microscope) with Zeiss LSM image examiner software. Cells were imaged using a Zeiss Plan-Apo $20 \times / 1.3$ objective lens with a 488 -mm laser for excitation and 505-mm pan filter for emission.

\section{Platelet immunoblot analysis}

The preparation of platelet extracts, quantification of the total protein concentration and the immunoblot analysis were done as previously described. ${ }^{11}$ Blots were revealed with a polyclonal $\beta$-tubulin antibody (SanverTECH, Boechout, Belgium), a polyclonal Gq $\alpha$ antibody (SanverTECH) or a polyclonal $\beta 1$-tubulin specific antibody (provided by $\mathrm{Dr}$ Sally Lewis and Dr Nicholas Cowan, New York University, New York). ${ }^{12}$ The secondary antibody was conjugated with horseradish peroxidase (HRP) and staining was performed with the Western blotting enhanced chemiluminescence (ECL) detection reagent (Amersham Biosciences, Uppsala, Sweden).

\section{Human platelet aggregation, secretion, and shape change}

Blood was anticoagulated with $3.8 \%$ (wt/vol) trisodium citrate (9:1) and the platelet count in the PRP was adjusted to $250 \times 10^{9}$ platelets/L. Platelet aggregation was performed on 2 dual-channel Chrono-Log Aggregometers (Chronolog, Havertown, PA), by simultaneously recording 4 tracings. Platelet secretion was determined by measuring the release of adenosine triphosphate (ATP) using luciferin/luciferase reagent (Kordia, Leiden, the Netherlands). Platelet aggregation and secretion were recorded in real time at $37^{\circ} \mathrm{C}$ with stirring $(1000 \mathrm{rpm})$ after stimulation with Horm collagen, adenosine diphosphate (ADP; Sigma Chemical), epinephrine, thrombin receptor activating peptide (TRAP; Peninsula Laboratories Europe, Belmont, CA), or the thromboxane A2 (TXA2) analog U46619 (Sigma Chemical). For studying platelet shape change response, tirofiban (2.5 $\mu \mathrm{g} / \mathrm{mL}$; Aggrastat, Merck Sharp \& Dome, Vienna, Austria), a glycoprotein $\mathrm{II}_{\mathrm{b}} \mathrm{III}_{\mathrm{a}}$ receptor antagonist, was added prior to agonist addition. Experiments were repeated using blood obtained from 5 unrelated Q43P $\beta 1$-tubulin carriers and each time a different control donor.

\section{Flow chamber and perfusion studies}

Platelet interaction with immobilized Horm collagen $(200 \mu \mathrm{g} / \mathrm{mL})$ was studied under flow conditions producing a wall shear rate of $600 \mathrm{~s}^{-1}$ or 300 $\mathrm{s}^{-1}$ in a parallel plate flow chamber essentially as described. ${ }^{13}$ The coverslip with immobilized collagen constituted the bottom of the chamber, and the actual chamber was formed by a $254-\mu \mathrm{m}$ high silicon rubber gasket designed with a conically shaped flow path, thus resulting in a 3 -fold increase of wall shear rate from the inlet of the chamber to the outlet. Heparinized blood was perfused at $37^{\circ} \mathrm{C}$ with an inverted syringe pump (Harvard Instruments, South Natick, MA) at a flow rate of $1 \mathrm{~mL} / \mathrm{min}$. After each perfusion run, the coverslips were removed from the perfusion chambers, rinsed with PBS, fixed in glutardialdehyde $(0.5 \% 10 \mathrm{mM}$ sodium phosphate, $150 \mathrm{mM} \mathrm{NaCl}, \mathrm{pH}$ 7.4), and stained with May-GrünwaldGiemsa. Platelet adhesion was quantified with a light microscope (Leica DM RBE; Leica, Wetzlar, Germany) equipped with a charge-coupled device (CCD) camera. Experiments were repeated using blood obtained from 3 unrelated Q43P $\beta 1$-tubulin carriers and 2 different control donors. The surface area coverage (percentage) was analyzed by the Java image processing program ImageJ 1.34g (National Institutes of Health, Bethesda, MD).

\section{Population study}

The $\beta 1$-tubulin gene was first studied in 33 unrelated white patients with unexplained congenital thrombocytopenia. We then performed genotypic analysis on 272 unrelated controls, comprising 269 Flemish and 3 Chinese subjects, who remained free from cardiovascular disease throughout this study. These 272 controls consisted of 180 bone marrow donors and 89 blood donors. Additionally, we studied 573 samples from a cohort of white patients with cardiovascular disease. A selection was made from all patients who had been referred to the ambulatory cardiac rehabilitation program of the University Hospitals of Leuven from 1990 through 2001 after myocardial infarction, percutaneous transluminal coronary angioplasty, coronary artery bypass grafting, stable angina pectoris that did not limit exercise performance, or a combination of these. Patients after heart transplantation, after implantation of a pacemaker, a cardioverter defibrillator or an artificial valve, or after any other cardiac surgery were excluded. Informed consent was obtained from all participants and/or their legal representatives. This study was approved by the Institutional Review Board of the University of Leuven.

\section{Statistical analyses}

GraphPad InStat 3.01 software (GraphPad Software, San Diego, CA) was used. Continuous variables with little to mild skewness were summarized as means $\pm \mathrm{SD}$ and compared by means of the Student $t$ test. Discrete variables were represented as frequencies and group percentages. Nominal variables were tested with the Pearson $\chi^{2}$ test. A $2 \times 2$ contingency table 
analysis with Fisher exact test was applied for the case-control study. Two-sided $P$ values less than .05 were considered significant. Results are given as odds ratios with $95 \%$ confidence intervals.

\section{Results}

\section{Identification of the Q43P variant in human platelet $\beta 1$-tubulin}

Studies in $\beta 1$-tubulin deficient mice showed that the absence of this $\beta$-tubulin isoform in platelets leads to the development of thrombocytopenia and spherocytic platelets. ${ }^{7,10}$ We were interested whether mutations in the human $\beta 1$-tubulin gene could account for the platelet maturation and functional defect in 33 unrelated patients with an undefined congenital macrothrombocytopenia (Table 1). All patients had an isolated nonimmune thrombocytopenia with a platelet count of lesss than $150 \times 10^{9}$ platelets/L blood, except patient 23 and patient 3 , who only have a mild thrombocytopenia but enlarged platelets. By thorough clinical and genetic analysis, we could exclude congenital amegakaryocytic thrombocytopenia (CAMT), the Bernard Soulier syndrome, the DiGeorge syndrome/ velocardiofacial syndrome (22q11 deletion), the Wiskott-Aldrich syndrome, GATA1 deficiency, and the A156V mutation (Bolzano variant) in GP1BA. ${ }^{11,14}$ Electron microscopy was performed on platelets from all thrombocytopenic patients and also from the patients' parents carrying the Q43P $\beta 1$-tubulin variant (Table 1 and Figure S1; see the Supplemental Figure link at the top of the online article, at the Blood website) and showed enlarged round platelets with ultrastructural abnormalities such as the presence of GATA1like inclusions in most of them. ${ }^{11}$

We have sequenced TUBB1 from platelet RNA of a thrombocytopenic patient (patient 2, Table 1) with spherocytic platelets and found a heterozygous $\mathrm{AG}>\mathrm{CC}$ substitution (Figure 1A). This mutation causes a $\mathrm{Q} 43 \mathrm{P}$ amino acid substitution and removes a restriction enzyme site for $P v u I I$ (Figure 1B). Sequence analyzed confirmed that the same heterozygous double-nucleotide substitution was present in 8 (4 women and 4 men; 24.2\%) of the 33 patients with macrothrombocytopenia (Table 1) and was the only variation in the coding region of the $\beta 1$-tubulin gene. However, since the inheritance of thrombocytopenia in these 8 families has autosomal recessive characteristics, with both parents having either a moderate thrombocytopenia or normal platelet count with an increased mean platelet volume (MPV), the $\beta 1$-tubulin variant by itself could not explain the more severe thrombocytopenia in these 8 patients. The $\beta 1$-tubulin substitution is located in a residue that is highly conserved in all $\beta$-tubulin isoforms of different species (Figure 1C).

To further assess the contribution of the Q43P $\beta 1$-tubulin mutation on platelet maturation and/or function, we investigated whether this variant is also present in the general population. Of 272 unrelated healthy controls, 29 (10.6\%) had the heterozygous $\beta 1$-tubulin substitution and 6 ( 5 white women and 1 Chinese man) of them were prepared to participate in further platelet functional studies (Table 1). These heterozygous carriers have a normal platelet count and a relatively high MPV. All other controls were anonymous donors or could not give blood for other reasons, such as use of medication. Sequence analysis confirmed the presence of the same double-nucleotide substitution as earlier found in the patients with thrombocytopenia.

\section{The effect of the Q43P $\beta$ 1-tubulin mutation} on platelet morphology

All Q43P $\beta$ 1-tubulin carriers had a normal platelet count, but electron microscopy performed on platelets of $3 \mathrm{Q} 43 \mathrm{P}$ carrier platelets showed the presence of enlarged and rounder platelets compared with the discoid shape of platelets from control subjects (Figure 2A-B), as was also seen in $\beta 1$-tubulin-deficient mice. ${ }^{7}$ These abnormal round platelets (about $50 \%$ of all platelets) have a disturbed marginal band of microtubules and present with organellefree zones, centralized platelet granules, and sometimes abnormal membrane complexes.

Immunoblot analysis showed reduced levels of both $\beta 1$-tubulin and the total $\beta$-tubulin fraction in platelet lysates from the 6 heterozygous Q43P carriers (Figure 3A). Therefore, the mutant seems to have a totally different effect on the $\beta$-tubulin organization than the defect originally described in $\beta 1$-tubulin knock-out mice. These mice do not express $\beta 1$-tubulin, but the overall $\beta$-tubulin fraction in their platelets is not reduced because of a compensatory $\beta 2$ - and $\beta 5$-tubulin overexpression. ${ }^{7}$ The overall reduced $\beta$-tubulin levels in platelets from the heterozygous $\beta 1$ tubulin carriers could be explained by the fact the Q43P $\beta 1$-tubulin isoform incorporates into microtubules at a lower efficiency than does the wild-type $\beta 1$-tubulin. Indeed, when human megakaryocytic MEG01 cells were transfected with a GFP-tagged $\beta 1$-tubulin, the Q43P variant showed reduced levels of a delocalized GFP signal, indicative of a defective integration of the mutant in the endogenous $\beta$-tubulins (Figure 3B). Spreading of these transfected MEG01 cells on a collagenor fibrinogen-coated surface clearly demonstrated the disturbed marginal band in the presence of the Q43P substitution (Figure 3B). Transfection studies in HEK293 cells showed the same delocalization with the Q43P variant (data not shown).

\section{The effect of the Q43P $\beta 1$-tubulin mutation on platelet function}

Cytoskeletal elements determine the changes in platelet cell shape, which occur during adhesion, aggregation, and release of granular contents as part of the activation process. In contrast to the minor effect of $\beta 1$-tubulin deficiency on platelet function in mice, the heterozygous Q43P $\beta 1$-tubulin platelets show a defective aggregation, secretion, and adhesion. PRP prepared from 5 heterozygous Q43P $\beta 1$-tubulin carriers was functionally tested by aggregation. A concentration of $1.5 \mu \mathrm{M}$ TRAP induced a weak and reversible aggregation, as shown in Figure 4A. Their platelets responded well to other agonists (ADP, arachidonic acid, ristocetin, and epinephrine), except for a somewhat retarded collagen aggregation. The platelet shape change was measured after activation of PRP with ADP $(5 \mu \mathrm{M})$, collagen $(2 \mu \mathrm{g} / \mathrm{mL})$, or TRAP $(2 \mu \mathrm{M})$ in the presence of a $\mathrm{GPI}_{\mathrm{b}} \mathrm{II}_{\mathrm{a}}$ inhibitor to exclude platelet aggregation. Previous studies indicate that changes in the level of tubulin polymerization correlate with the transition from discoid to spheric morphology. ${ }^{15}$ We found no significant differences in the shape change between platelets from Q43P carriers and controls (data not shown). Although the platelets from Q43P $\beta 1$-tubulin carriers have a normal amount of alpha and dense granules, these are more centralized and clustered, with simultaneous presence of organelle-free zones. Following stimulation of PRP with relatively low concentrations of ADP $(5 \mu \mathrm{M})$ or collagen $(2 \mu \mathrm{g} / \mathrm{mL}), \mathrm{Q} 43 \mathrm{P} \beta 1$-tubulin carriers show a significantly decreased ATP release (Figure 4B). These differences were not seen using a higher concentration of these agonists $(20 \mu \mathrm{M}$ ADP and $5 \mu \mathrm{g} / \mathrm{mL}$ collagen).

Studies describing platelet adhesion to glass have shown that this process is composed of a close spatial and functional relation between actin filaments, myosin molecules, and microtubules. ${ }^{16}$ To evaluate the relation between the Q43P $\beta 1$-tubulin variant in platelet microtubules and platelet adhesion to collagen fibers, heparinized blood from 3 Q43P $\beta 1$-tubulin carriers was perfused over collagen under high-shear flow conditions. Platelets from Q43P $\beta 1$-tubulin carriers showed a reduced thrombus formation on collagen under flow when compared with control platelets (Figure 5A-B). 
From www.bloodjournal.org at kuleuven on October 29, 2009. For personal use only.

Table 1. Characteristics of patients with inherited thrombocytopenia with or without the Q43P $\beta$ 1-tubulin substitution and of the Q43P $\beta 1$-tubulin carriers in a healthy population

\begin{tabular}{|c|c|c|c|c|c|c|c|}
\hline \multirow[b]{2}{*}{ Subjects } & \multirow[b]{2}{*}{ Sex } & \multirow{2}{*}{$\begin{array}{c}\text { Platelet } \\
\text { count, } \\
\times 10^{9} / \mathrm{L}, \\
150-44 \S\end{array}$} & \multirow{2}{*}{$\begin{array}{c}\text { Mean } \\
\text { platelet } \\
\text { volume, } \\
\mathrm{fL}, 8-12.5 \S\end{array}$} & \multicolumn{3}{|c|}{ Platelet function studies } & \multirow[b]{2}{*}{ Platelet electron microscopy } \\
\hline & & & & $\begin{array}{l}\text { Clinical } \\
\text { bleeding } \\
\text { problem }\end{array}$ & $\begin{array}{c}\text { Decreased } \\
\text { collagen } \\
\text { aggregation }\end{array}$ & $\begin{array}{c}\text { Decreased } \\
\text { ATP } \\
\text { secretion }\end{array}$ & \\
\hline \multicolumn{8}{|c|}{$\begin{array}{l}\text { Q43P } \beta \text { 1-tubulin } \\
\text { thrombocytopenia patients* }\end{array}$} \\
\hline Patient 1 & M & 117 & $>15$ & - & + & + & Enlarged, round platelets, inclusions \\
\hline Parent 1 & $\mathrm{~F}$ & 163 & 13.3 & - & + & + & Enlarged, round platelets, inclusions $\ddagger$ \\
\hline Patient 2 & M & 110 & $>15$ & + & + & + & Enlarged, round platelets, inclusions $\ddagger$ \\
\hline Parent 2 & $\mathrm{~F}$ & 281 & 12.8 & - & + & + & Enlarged, round platelets, inclusion $\ddagger$ \\
\hline Patient 3 & $\mathrm{~F}$ & 85 & 13.3 & + & + & + & Enlarged, round platelets \\
\hline Parent 3† & M & NA & NA & - & NA & NA & NA \\
\hline Patient 4 & M & 33 & $>15$ & - & NA & NA & Enlarged, round platelets, inclusions \\
\hline Parent 4 & $\mathrm{~F}$ & 46 & $>15$ & - & NA & NA & Enlarged, round platelets, inclusions \\
\hline Patient 5 & M & 70 & $>15$ & + & + & NA & Enlarged, round platelets, inclusions \\
\hline Parent 5 & $\mathrm{~F}$ & 214 & 13.4 & - & NA & NA & Enlarged, round platelets, inclusions $\ddagger$ \\
\hline Patient 6 & $\mathrm{~F}$ & 144 & 13.2 & + & NA & NA & Enlarged, round platelets, inclusions \\
\hline Patient 7 & $\mathrm{~F}$ & $<150$ & $>15$ & + & NA & NA & NA \\
\hline Patient 8 & $\mathrm{~F}$ & 102 & 11.7 & + & + & + & Enlarged, round platelets, inclusions \\
\hline \multicolumn{8}{|c|}{$\begin{array}{l}\text { Q43 } \beta 1 \text {-tubulin } \\
\text { thrombocytopenia patients }\end{array}$} \\
\hline Patient 9 & $\mathrm{~F}$ & 66 & $>15$ & + & NA & NA & Enlarged, round platelets, inclusions \\
\hline Patient 10 & $\mathrm{~F}$ & 50 & $>15$ & + & NA & NA & Enlarged platelets \\
\hline Patient 11 & $\mathrm{~F}$ & 81 & $>15$ & - & - & NA & $\begin{array}{l}\text { Enlarged, round platelets, small dense granules } \\
\text { and inclusions }\end{array}$ \\
\hline Patient 12 & $\mathrm{~F}$ & 87 & $>15$ & + & NA & NA & Giant, round platelets and many inclusions \\
\hline Patient 13 & $\mathrm{~F}$ & 59 & $>15$ & - & - & - & $\begin{array}{l}\text { Enlarged platelets, lacking granules and with } \\
\text { many inclusions }\end{array}$ \\
\hline Patient 14 & $\mathrm{~F}$ & 90 & $>15$ & + & + & + & Giant platelets \\
\hline Patient 15 & $\mathrm{~F}$ & 36 & $>15$ & - & + & + & Enlarged platelets with few dense granules \\
\hline Patient 16 & $\mathrm{~F}$ & 90 & $>15$ & - & - & - & Enlarges platelets with few inclusions \\
\hline Patient 17 & M & 26 & $>15$ & - & - & - & NA \\
\hline Patient 18 & M & 67 & $>15$ & NA & NA & NA & NA \\
\hline Patient 19 & $\mathrm{~F}$ & 103 & $>15$ & + & NA & + & Enlarged platelets with few inclusions \\
\hline Patient 20 & $\mathrm{~F}$ & 140 & 13.7 & + & + & + & Enlarged platelets, abnormal alpha granules \\
\hline Patient 21 & $\mathrm{~F}$ & 60 & 10.2 & + & + & + & Enlarged, round platelets \\
\hline Patient 22 & M & 66 & 10.5 & NA & NA & NA & Enlarged, round platelets \\
\hline Patient 23 & $\mathrm{~F}$ & 174 & 11.8 & - & - & - & Enlarged platelets, abnormal alpha granules \\
\hline Patient 24 & $\mathrm{~F}$ & 53 & $>15$ & - & NA & NA & Giant platelets, abnormal alpha granules \\
\hline Patient 25 & $\mathrm{~F}$ & 57 & 14.0 & - & NA & NA & Enlarged platelets \\
\hline Patient 26 & M & 69 & $>15$ & - & - & - & Giant platelets \\
\hline Patient 27 & $\mathrm{~F}$ & 100 & 13.3 & + & + & NA & Enlarged platelets, abnormal dense granules \\
\hline Patient 28 & M & 84 & $>15$ & NA & + & NA & Enlarged, round platelets with inclusions \\
\hline Patient 29 & M & 101 & $>15$ & - & NA & NA & $\begin{array}{l}\text { Enlarged, round platelets with inclusions and } \\
\text { few alpha granules }\end{array}$ \\
\hline Patient 30 & M & 75 & $>15$ & + & + & NA & Giant platelets \\
\hline Patient 31 & M & 171 & 13.2 & - & - & - & $\begin{array}{l}\text { Enlarged, round platelets, many dense granules } \\
\text { and inclusions }\end{array}$ \\
\hline Patient 32 & M & 107 & 12.9 & - & - & - & Giant platelets with abnormal granules \\
\hline Patient 33 & M & 56 & $>15$ & - & - & - & Giant platelets \\
\hline \multicolumn{8}{|c|}{$\begin{array}{l}\text { Q43P } \beta \text { 1-tubulin healthy } \\
\text { population }\end{array}$} \\
\hline Carrier 1 & $\mathrm{~F}$ & 230 & 10.8 & - & NA & + & Enlarged, round platelets with inclusions \\
\hline Carrier 2 & $\mathrm{~F}$ & 284 & 11.6 & - & + & + & Enlarged, round platelets with inclusions \\
\hline Carrier 3 & M & 230 & 9.6 & - & + & + & NA \\
\hline Carrier 4 & $\mathrm{~F}$ & 195 & 11.4 & - & + & + & Enlarged, round platelets with inclusions \\
\hline Carrier 5 & $\mathrm{~F}$ & 268 & 9.7 & - & + & + & NA \\
\hline Carrier 6 & $\mathrm{~F}$ & 258 & 10.6 & - & + & + & NA \\
\hline
\end{tabular}

The presence $(+)$ or absence $(-)$ of clinical bleeding problems is indicated for all subjects. Bleeding problems are mostly associated with an increased Ivy bleeding time and include the presence of petechiae, epistaxis, and/or small superficial ecchymoses. A decreased collagen-induced $(0.5 \mu \mathrm{g} / \mathrm{mL})$ aggregation $(+)$ means an aggregation of only $50 \%$ or less. A decreased ATP secretion $(+)$ after collagen $(2 \mu \mathrm{g} / \mathrm{mL})$ activation means a value less than $3 \mu$ M ATP release. Normal collagen-induced aggregation and ATP secretion values are shown by the minus sign.

NA indicates not available.

${ }^{*}$ Sub-subentries in this category are parents of patients.

†Carrier RR is homozygous for the double $\beta 1$-tubulin nucleotide substitution.

‡Electron microscopy pictures of platelets from these individuals are available in Figure S1.

$\S$ Normal values for platelet count and mean platelet volume are indicated. 
A

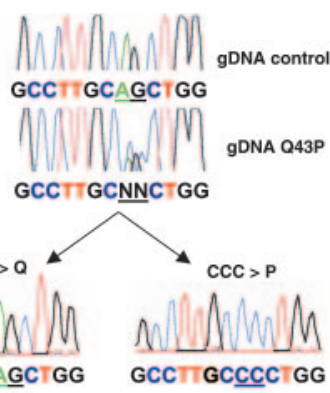

B

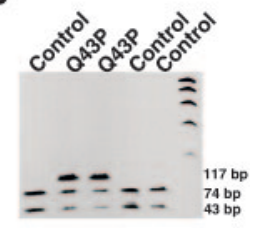

C

\begin{tabular}{|c|c|c|}
\hline \multirow[b]{2}{*}{ Human $\beta 1$-tubulin } & \multicolumn{2}{|r|}{ Q43P } \\
\hline & AGSDRGA & SALQLERISVYY NEAYG \\
\hline Human $\beta 4$-tubulin & $-\mathrm{G}=-\mathrm{G}-\mathrm{S}$ & - LQL- RISVYY NEA-G \\
\hline Human $\beta 5$-tubulin & $-\mathrm{G}--\mathrm{G}-\mathrm{S}$ & - LQLE RISVYY NEA-- \\
\hline Mouse $\beta 1$-tubulin & AGS --G-S & ALQLE RISVYY NEAYG \\
\hline Mouse $\beta 2$-tubulin & $-\mathrm{G}=-\mathrm{G}-\mathrm{S}$ & - LQLERI-VYY NEA-G \\
\hline Mouse $\beta 3$-tubulin & $-\mathrm{G}--\mathrm{G}-\mathrm{G}$ & - LQLE RISVYY NEA-- \\
\hline Chicken $\beta 6$-tubulin & AG- - G-- & - LQLERI-VY- NEAY- \\
\hline Chicken $\beta 2$-tubulin & -G S -G-S & - LQLERI-VYY NEAY- \\
\hline Pig $\beta$-tubulin & $-\mathrm{G}$ S--G-S & - LQLERI-VYY NEA-G \\
\hline rosophila $\beta$-tubulin & $-\mathrm{G}---\mathrm{G}-\mathrm{S}$ & ALQ-E RI-VYY NEA-- \\
\hline Xenopus $\beta$-tubulin & $-\mathrm{G}=-\mathrm{G}-\mathrm{S}$ & - LQLERI-VYY NEA-G \\
\hline
\end{tabular}

Figure 1. Mutational analysis of $\beta 1$-tubulin. (A) Sequencing of $\beta 1$-tubulin cDNA from a control subject and a patient with thrombocytopenia (Q43P). A double base-pair substitution, AG to CC, was found at nucleotide positions 130 and 131 of the $\beta 1$-tubulin gene, as could easily be detected in subcloned $\beta 1$-tubulin reverse transcriptase (RT)-PCR fragments from this patient. (B) The region encompassing exon 2 (117-bp PCR product) was amplified from genomic DNA before allele genotyping using $P v u l l$ digestion (74 and $43 \mathrm{bp}$ ) and analysis by agarose gel electrophoresis. The CAG-to-CCC double substitution (Q43P) leads to a loss of $P$ vull digestion. (C) This mutation results in a replacement of the highly conserved Gln at position 43 for Pro.

\section{Clinical relevance of the Q43P $\beta$ 1-tubulin mutation}

Since the Q43P $\beta$ 1-tubulin variant reduces platelet aggregation, secretion, and adhesion, it may protect against cardiovascular disease. To test this hypothesis, a case-control study was performed; all population controls were white. The prevalence of the Q43P substitution in unrelated controls was $10.4 \%$ (28 of 269), while it was halved in patients with cardiovascular disease ( 30 of $573,5.2 \%$ versus $10.4 \% ; P<.001$ with $\mathrm{OR}=2.1 ; 95 \%$ CI 1.22-3.59). The characteristics of the patients with cardiovascular disease are listed in Table 2 by $\beta 1$-tubulin genotype. Further subgroup analysis by severity of coronary heart disease did not reveal any significant differences between the Q43P $\beta 1$-tubulin carriers and noncarriers (Table 2).

By analyzing the prevalence of the $\beta 1$-tubulin Q43P variant in the men and women of the case-control study separately, it becomes obvious that the $\beta 1$-tubulin functional variant only protects against cardiovascular disease in men. In men, the prevalence of the $\beta 1$-tubulin was $9.1 \%$ (11 of 120) in controls versus $4 \%$ (21 of 523) in patients with cardiovascular disease $(P=.03$ with an $\mathrm{OR}=2.41 ; 95 \%$ CI $1.13-5.1)$, while in women there was no difference: $11.4 \%$ (17 of 149 ) versus $14 \%$ ( 7 of 50 ; $P=.62$ with an $\mathrm{OR}=1.26 ; 95 \%$ CI.49-3.25).

\section{Discussion}

In this study, we were able to identify both structural and functional differences between wild-type $\beta 1$-tubulin and the Q43P variant that may contribute to the development of macrothrombocytopenia or act as a protective factor against cardiovascular disease. The Q43P $\beta 1$-tubulin variant results from a double nucleotide substitution $(\mathrm{AG}>\mathrm{CC})$. Two independent complex germ-line substitutions occurring at an identical site are far rarer than point mutations affecting single nucleotides. ${ }^{17}$ This polymorphism probably is a very ancient mutation relative to a unanimous ancestral primate allele. It is present at a relatively high frequency in the general white population, and we also detected this substitution in a Chinese individual.

Although inherited thrombocytopenias are relatively rare platelet disorders, their frequency is probably much underestimated because of diagnostic difficulties and a mostly subclinical phenotype. A number of studies using knock-out mouse models as well as the recently described genetic defects responsible for several inherited thrombocytopenias reveal the importance of certain genes in normal megakaryocyte proliferation, maturation, and platelet production. ${ }^{18-20}$ Studies in $\beta 1$-tubulin-deficient mice demonstrated that this megakaryocyte-specific $\beta$-tubulin isoform is necessary to permit normal proplatelet abundance and, independently, proved to be essential in the generation of the discoid platelet shape. ${ }^{7,10}$ In the present study, we describe the first human $\beta 1$-tubulin variant (Q43P), leading to a defective tubulin organization. Heterozygous carriers have spherocytic and enlarged platelets with abnormal membrane complexes and organelle-free zones in the cytoplasm. White and de Alarcon ${ }^{21}$ already described a platelet bleeding disorder, characterized by platelet spherocytosis. The Q43P $\beta 1$-tubulin variant is present in $10.6 \%$ of the general population but also in $24.2 \%$ of 33 unrelated patients with congenital macrothrombocytopenia. It is unlikely that this functional variant would be the only genetic factor contributing to the clinical problems in these patients, since in all these families the thrombocytopenia seems to be an autosomal recessive disorder. Furthermore, electron microscopy of platelets from some thrombocytopenia patients without the Q43P $\beta 1$-tubulin substitution also showed enlarged and round platelets. Molecular studies on the other platelet $\beta$-tubulin isoforms and the transcription factor nuclear

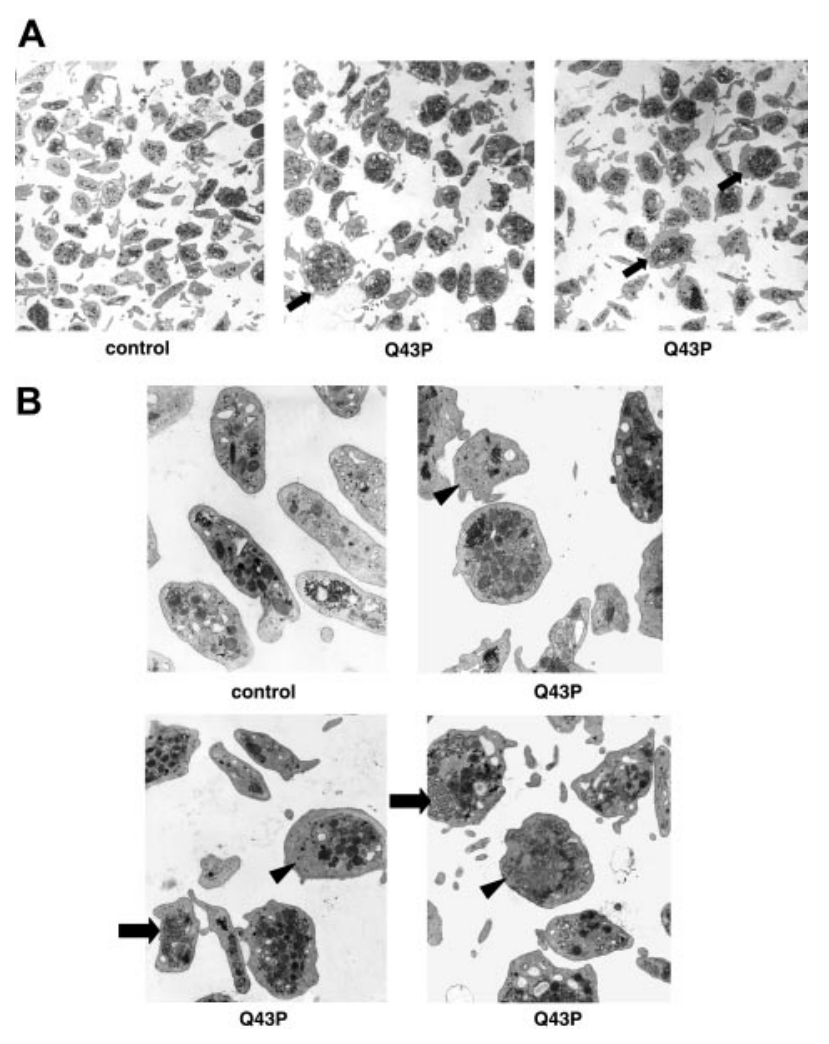

Figure 2. Ultrastructural analysis of platelets. (A) Electron microscopy of platelets (original magnification, $\times 7800$ ) of a healthy individual and 2 heterozygous $\beta 1$-tubulin Q43P carriers (carriers 1 and 2). Almost $50 \%$ of Q43P carrier platelets are enlarged and round (arrow). (B) Electron microscopy of platelets (original magnification, $\times 22500$ ) of a healthy individual and 3 heterozygous $\beta 1$-tubulin Q43P carriers, showing cytoplasmic inclusions composed of smooth endoplasmic reticulum and abnormal membrane complexes (arrow). Note also the organelle-free zones in some platelets (arrowhead). There is a normal number of more centralized platelet alpha and dense granules. 

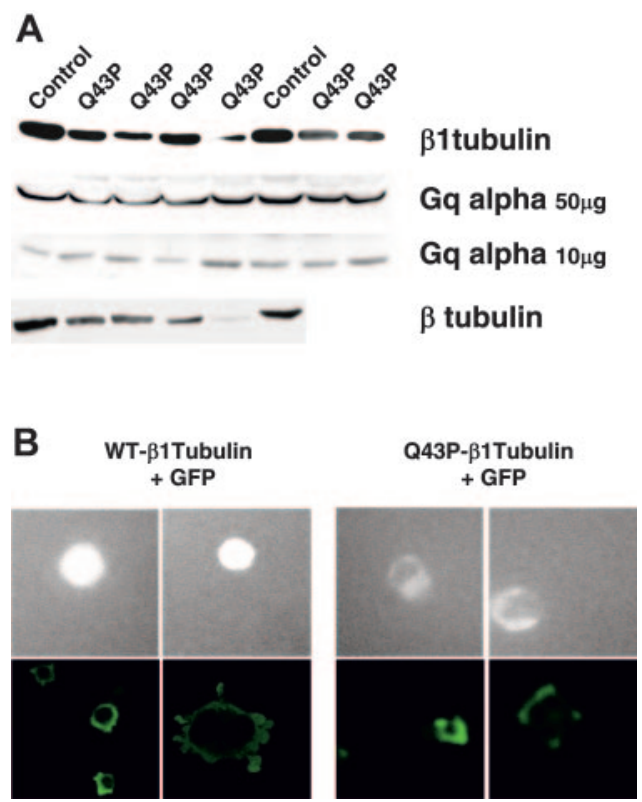

Figure 3. $\boldsymbol{\beta} 1$-tubulin protein expression. (A) Immunoblot analysis of $\beta 1$-tubulin, $\beta$-tubulin, and $\mathrm{Gq}$ alpha in the total platelet protein fractions (10 or $50 \mu \mathrm{g}$ ) from healthy controls and 4 to 6 unrelated heterozygous Q43P carriers. Gq alpha serves as a loading control. (B) Contrast microscopy of GPF-tagged $\beta 1$-tubulin in MEG01 megakaryocytic cells transfected with pEGFP-WT- $\beta 1$-tubulin or pEGFP-Q43P- $\beta 1$-tubulin (top 4 rows). Immunofluorescence analysis of transfected MEG01 cells on Horm collagen (bottom left panels) or fibrinogen (bottom right panels). Original magnification, $\times 20$.

factor-erythroid 2 (NF-E2) $)^{22}$ in all patients are ongoing. Previous studies have already shown that the expression of $\beta 1$-tubulin is completely abolished in NF-E2-deficient mouse megakaryocytes. ${ }^{6}$

The heterozygous carriers within the healthy population seem to have normal platelet numbers and a moderately elevated MPV compatible with the fact that electron microscopy on these individuals revealed 2 platelet populations consisting of about $50 \%$ normal discoid platelets and $50 \%$ enlarged rounder platelets. The normal ranges of the platelet count $\left(150-440 \times 10^{9} / \mathrm{L}\right)$ and MPV (8-12.5 fL) as determined by our hematologic counter are rather broad, but these calibration values were obtained in a healthy population also including $10.6 \%$ Q43P $\beta 1$-tubulin carriers. Heterozygous carrier platelets show a reduced platelet aggregation, secretion, and collagen adhesion, though platelet shape change was
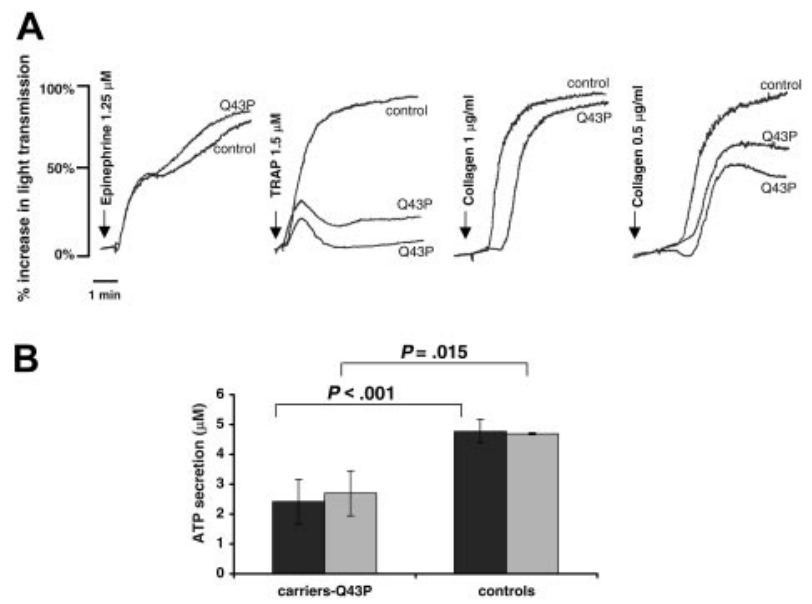

Figure 4. Platelet aggregation and secretion. (A) Platelet aggregations in citrated PRP from a control subject and 2 Q43P carriers (carriers 2 and 4) are shown with the indicated agonists and concentrations. Aggregations were performed in 5 unrelated carriers on separate occasions with similar results. (B) Platelet ATP secretion after stimulation of citrated PRP with ADP $(5 \mu \mathrm{M}, \square)$ or collagen $(2 \mu \mathrm{g} / \mathrm{mL}$, 圆) shows a significantly decreased response in the 6 unrelated Q43P carriers compared with 6 healthy controls.
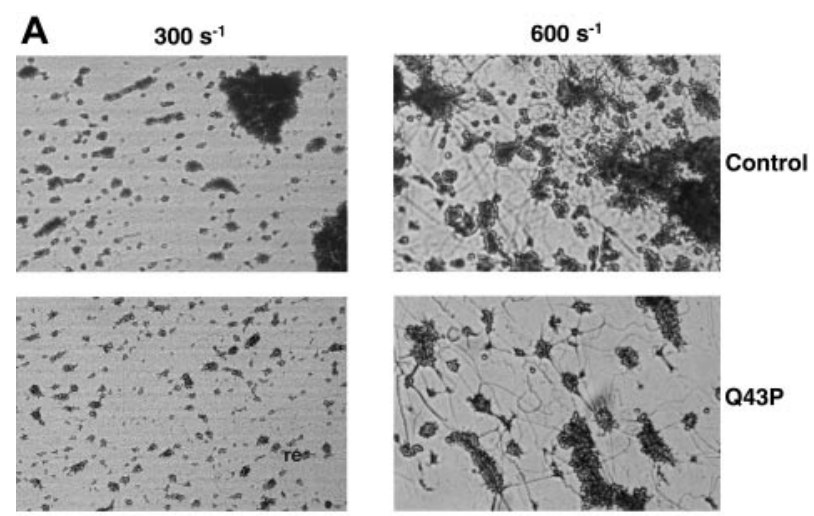

B

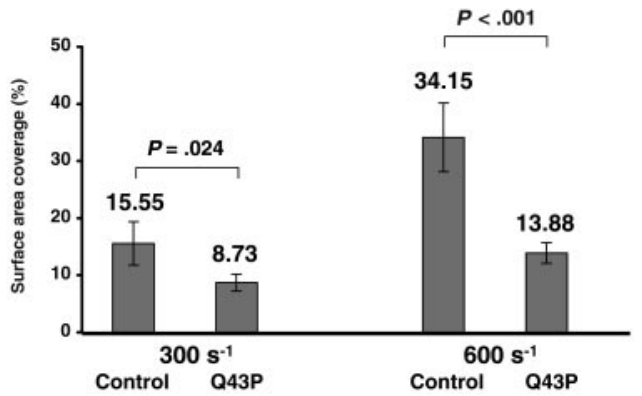

Figure 5. Platelet adhesion studies. Platelet adhesion to Horm collagen was studied by flow chamber experiments. Heparinized blood from 3 unrelated Q43P carriers (carriers 1, 5, and 6) and 2 control subjects was perfused over collagencoated slides at shear rates of 300 and $600 \mathrm{~s}^{-1}$. (A) The figures represent images of a portion of the flow chamber for platelets derived from the indicated subjects and show that the platelet aggregates are smaller in the Q43P carriers. Original magnification, $\times 200$. (B) Mean surface area coverage (percentage) of platelets at shear rates of 300 and $600 \mathrm{~s}^{-1}$ after a perfusion time of 5 minutes for the indicated subjects. The statistical significance of observed differences between the 2 groups is indicated above each pair of bars. Error bars indicate mean \pm standard deviation (SD).

not significantly different between carriers and noncarriers. Although nearly $50 \%$ of the platelets from carriers have a resting spherical shape, differences in shape change after stimulation of platelets with different agonists could not be observed. The effect of a homozygous Q43P $\beta 1$-tubulin substitution on platelet shape change or other functional studies could not yet be defined because only 1 homozygous subject was identified, who was not willing to participate in further studies.

While the heterozygous Q43P $\beta 1$-tubulin carriers have a reduced function, the $\beta 1$-tubulin-deficient mice present with only minor abnormalities in platelet hemostatic functions. Besides the fact that human platelets are more easy to handle and study in detail than mouse platelets, this can probably be explained by the fact that the loss of $\beta 1$-tubulin expression in mouse platelets was overcome by overexpression of the other platelet $\beta$-tubulin variants, ${ }^{7}$ while the Q43P carrier platelets not only show reduced $\beta 1$-tubulin but also total $\beta$-tubulin protein levels. In addition, incorporation of GFP-tagged Q43P $\beta$ 1-tubulin into wild-type tubulin structures seems to be inefficient and delocalized.

Due to the platelet dysfunction phenotype, the Q43P $\beta 1$-tubulin variant could not only be conceived as a genetic risk factor for the development of thrombocytopenia but also as a protective genetic factor against cardiovascular disease. Indeed, a case-control study showed that the prevalence of Q43P $\beta 1$-tubulin carriership was lower in patients with atherosclerotic cardiovascular disease than in the general population. Most platelet gene polymorphisms studied to date as risk factors for cardiovascular disease were described in the platelet-specific glycoprotein receptors GPIb/IX/V, GPIa/IIa, and GPIIb/IIIa, ${ }^{23,24}$ but to our knowledge never in genes important 
Table 2. Characteristics of patients with cardiovascular disease by $\beta 1$-tubulin genotype

\begin{tabular}{|c|c|c|c|}
\hline & Noncarriers $\dagger$ & $\begin{array}{c}\text { Heterozygous } \\
\text { 及1-tubulin } \\
\text { Q43P } \\
\text { carriers } †\end{array}$ & $\boldsymbol{P}$ \\
\hline No. & 543 & 30 & NA \\
\hline Age, $y$ & $56.6 \pm 9.2$ & $53.8 \pm 9.0$ & .10 \\
\hline Female, \% & 9.1 & 23.3 & $.02 *$ \\
\hline Body mass index, $\mathrm{kg} / \mathrm{m}^{2}$ & $25.5 \pm 3.4$ & $24.6 \pm 3.4$ & .15 \\
\hline Triglyceride level, $\mathrm{mM}$ & $1.93 \pm 1.19$ & $1.61 \pm .91$ & .15 \\
\hline Cholesterol level, mM & $5.56 \pm 1.11$ & $5.67 \pm 1.25$ & .61 \\
\hline History of smoking, \% & 71.1 & 83.4 & .22 \\
\hline Diabetes mellitus, \% & 6.1 & 0.0 & .32 \\
\hline Hypertension, \% & 28.4 & 10.0 & $.04^{*}$ \\
\hline Family history, \% & 20.0 & 20.0 & .99 \\
\hline Myocardial infarction, \% & 68.5 & 70.0 & .86 \\
\hline Unstable angina, \% & 4.3 & 6.7 & .86 \\
\hline Coronary artery bypass graft, $\%$ & 38.2 & 46.7 & .45 \\
\hline Coronary angioplasty, $\%$ & 58.3 & 50.0 & .48 \\
\hline \multicolumn{4}{|l|}{ Effect of medication } \\
\hline Anticoagulation drugs, $\%$ & 5.5 & 6.7 & .79 \\
\hline Antiplatelet drugs, $\%$ & 88.2 & 90.0 & .99 \\
\hline Beta blockers, $\%$ & 84.3 & 83.3 & .88 \\
\hline Lipid-lowering drugs, \% & 16.4 & 30.0 & .09 \\
\hline Ace inhibitors, \% & 27.7 & 13.3 & .13 \\
\hline Coronary heart disease, no. & 272 & 14 & NA \\
\hline Single-vessel disease, $\%$ & 25 & 28.5 & .65 \\
\hline Two-vessel disease, $\%$ & 25.7 & 28.5 & .65 \\
\hline Three-vessel disease, $\%$ & 49.3 & 43.0 & .65 \\
\hline
\end{tabular}

Test for differences between groups with $\chi^{2}$ or $t$ test.

NA indicates not applicable.

*Two-sided $P$ values less than .05 were considered significant.

†Values are mean \pm SD or percentage of individuals.

for the platelet cytoskeleton organization. We believe our data may also speak to the issue of sexual dimorphism with respect to cardiovascular disease. Many studies have already shown that the incidence of cardiovascular disease differs between men and women, in part because of differences in risk factors and hormones. ${ }^{25-27}$ In contrast to our study describing the Q43P $\beta 1$-tubulin variant as a protective factor against cardiovascular disease in men, most studies have focused on studying risk factors for this disease in men. Although our functional assays studying the effect of the Q43P $\beta 1$-tubulin variant as inhibitor of platelet aggregation, secretion and adhesion showed no obvious differences in heterozygous male or female controls, further studies are needed to explain the sex difference for this functional polymorphism in relation with cardiovascular disease. Additional studies are needed to define whether the protective effect of the Q43P $\beta 1$-tubulin variant is negatively influenced by hormones or additional genetic factors in women. Since the Q43P $\beta 1$-tubulin variant seems to protect against arterial thrombosis by inhibiting platelet function, this protective effect may also be translated in a potential bleeding diathesis in these carriers. Although none of the 6 Q43P $\beta 1$-tubulin carriers from the healthy population or any of the patients' parents with the substitution had obvious clinical problems, this topic deserves further investigation.

In conclusion, the first described human $\beta 1$-tubulin substitution is located in a highly conserved residue and has major implications for platelet morphology and function. Additional studies including more patients and controls should be performed in other populations to confirm our initial results that the Q43P $\beta 1$-tubulin variant is a protective genetic factor against cardiovascular disease. On the other hand, its contribution to the development of thrombocytopenia should also be further studied in detail.

\section{Acknowledgments}

K.F. is a postdoctoral fellow, C.V.G. is the holder of a clinicalfundamental research mandate, and J.D. is Research Assistant of the Fund for Scientific Research-Flanders (F.W.O.-Vlaanderen, Belgium). We thank Dr Lewis and Dr Cowan for generously providing a $\beta 1$-tubulin specific antibody.

\section{References}

1. Sullivan KF. Structure and utilization of tubulin isotypes. Annu Rev Cell Biol. 1988;4:687-716.

2. Cleveland DW. The multitubulin hypothesis revisited: what have we learned? J Cell Biol. 1987;104:381-383.

3. Luduena RF. Multiple forms of tubulin: different gene products and covalent modifications. Int Rev Cytol. 1998;178:207-275.

4. Luduena RF. Are tubulin isotypes functionally significant? Mol Biol Cell. 1993;4:445-457.

5. Wang D, Villasante A, Lewis SA, Cowan NJ. The mammalian beta-tubulin repertoire: hematopoietic expression of a novel, heterologous betatubulin isotype. J Cell Biol. 1986;103:1903-1910.

6. Lecine P, Italiano JE Jr, Kim SW, Villeval JL, Shivdasani RA. Hematopoietic-specific beta 1 tubulin participates in a pathway of platelet biogenesis dependent on the transcription factor NF-E2. Blood. 2000;96:1366-1373.

7. Schwer HD, Lecine P, Tiwari S, Italiano JE Jr, Hartwig JH, Shivdasani RA. A lineage-restricted and divergent beta-tubulin isoform is essential for the biogenesis, structure and function of blood platelets. Curr Biol. 2001;11:579-586.

8. Radley JM, Hartshorn MA. Megakaryocyte fragments and the microtubule coil. Blood Cells. 1987; 12:603-614.

9. Italiano JE Jr, Lecine P, Shivdasani RA, Hartwig $\mathrm{JH}$. Blood platelets are assembled principally at the ends of proplatelet processes produ $120 \mathrm{ced}$ by differentiated megakaryocytes. J Cell Biol. 1999;147:1299-1312.

10. Italiano JE Jr, Bergmeier W, Tiwari S, et al.
Mechanisms and implications of platelet discoid shape. Blood. 2003;101:4789-4796.

11. Freson K, Devriendt K, Matthijs G, et al. Platelet characteristics in patients with $X$-linked macrothrombocytopenia because of a novel GATA1 mutation. Blood. 2001;98:85-92.

12. Lewis $\mathrm{SA}, \mathrm{Gu}$ W, Cowan NJ. Free intermingling of mammalian beta-tubulin isotypes among functionally distinct microtubules. Cell. 1987;49:539-548.

13. Theilmeier G, Lenaerts T, Remacle C, Collen D, Vermylen J, Hoylaerts MF. Circulating activated platelets assist THP-1 monocytoid/endothelial cell interaction under shear stress. Blood. 1999;94:2725-2734.

14. Savoia A, Balduini CL, Savino M, et al. Autosoma dominant macrothrombocytopenia in Italy is most frequently a type of heterozygous Bernard-Soulier syndrome. Blood. 2001;97:1330-1335.

15. Paul BZ, Kim S, Dangelmaier C, et al Dynamic regulation of microtubule coils in ADP-induced platelet shape change by p160ROCK (Rho-kinase). Platelets. 2003;14:159-169.

16. Cerecedo D, Stock R, Gonzalez S, Reyes E, Mondragon R. Modification of actin, myosin and tubulin distribution during cytoplasmic granule movements associated with platelet adhesion. Haematologica. 2002;87:1165-1176.

17. Smith NG, Webster MT, Ellegren H. A low rate of simultaneous double-nucleotide mutations in primates. Mol Biol Evol. 2003;20:47-53.

18. Van Geet C, Freson K, De Vos R, Vermylen J. Hereditary thrombocytopenias. In: Gresele $P$, Page CP, Fuster V, Vermylen J, eds. Platelets in Thrombotic and Non-Thrombotic Disorders:
Pathophysiology, Pharmacology and Therapeutics. Cambridge University Press, Cambridge, United Kingdom; 2002:515-527.

19. Drachman JG. Inherited thrombocytopenia: when a low platelet count does not mean ITP. Blood. 2004;103:390-398.

20. Schulze H, Shivdasani RA. Molecular mechanisms of megakaryocyte differentiation. Semin Thromb Hemost. 2004;30:389-398.

21. White JG, de Alarcon PA. Platelet spherocytosis: a new bleeding disorder. Am J Hematol. 2002;70: 158-166.

22. Shivdasani RA, Rosenblatt MF, Zucker-Franklin $D$, et al. Transcription factor NF-E2 is required for platelet formation independent of the actions of thrombopoietin/MGDF in megakaryocyte development. Cell. 1995;81:695-704.

23. Meisel C, Lopez JA, Stangl K. Role of platelet glycoprotein polymorphisms in cardiovascular diseases. Naunyn Schmiedebergs Arch Pharmacol. 2004;369:38-54

24. Kunicki TJ, Nugent DJ. The influence of platelet glycoprotein polymorphisms on receptor function and risk for thrombosis. Vox Sang. 2002;83:85-90.

25. Patel H, Rosengren A, Ekman I. Symptoms in acute coronary syndromes: does sex make a difference? Am Heart J. 2004 ;148:27-33.

26. Leinwand LA. Sex is a potent modifier of the cardiovascular system. J Clin Invest. 2003;112:302-307.

27. Devon HA, Zerwic JJ. Symptoms of acute coronary syndromes: are there gender differences? A review of the literature. Heart Lung. 2002;31:235-245. 